\title{
Strain resistors method test the magnetostrictive property of FebacCu1Nb3Si15.5B7/Silicone rubber compound film
}

\author{
Fu Yuan ${ }^{1, a^{*}}$, Guo Wei, ${ }^{2,}$, Cheng Xiang ping ${ }^{1}$, Wang $\mathrm{De}^{1}$ \\ ${ }^{1}$ Applied Physics Institute for Jiang Xi academy of sciences, NanChang 330028, China \\ ${ }^{2}$ JiangXi Key laboratory of Cu-W alloy, NanChang 330030, China \\ afu-yuan@hotmail.com, b124291713@qq.com
}

\section{Keywords: Magnetostriction, Amorphous powder, Silicone rubber, FebacCu1Nb3Si15.5B7}

\begin{abstract}
It is researched on the magnetostriction of FebacCu1Nb3Si15.5B7 amorphous powder and silicon rubber composite films in which the average particle size is about $20 \mu \mathrm{m}$ with different mass fractions. And also have determined the internal relationship between the composite ratio of powder and silicon rubber and the magnetostriction properties of the film. Meanwhile, in terms of generalized variational principle, the ranges of elastic modulus, Poisson's ratio and inner stress in the composite film can also be defined. Result shows: The mass ratio of FebacCu1Nb3Si15.5B7 amorphous powder and silicon rubber at 3:1, magnetostrictive coefficient is of a maximum value $2.1 \times 10-6$. Experimental result indicated that, the composite film is a composite material, which have wonderful supple magnetostriction property, and a nice material for making supple force senor.
\end{abstract}

\section{Introduction}

The magnetostriction property of FebacCu1Nb3 Si15.5B7 /Silicone rubber compound film is not only have business on resin matrix, mechanical property, and mass fraction of these two types of material, but also have more things to do with its productive technology and fabrication process. In current, stress sensitivity films have become a hot researching issues, which can be divided into piezomagnetic type and piezoelectric type. At present, piezoelectric films have been commonly used, while piezomagnetic films are infrequently covered. Favorable piezomagnetic stress sensitivity films should have two characteristics: 1 Favorable and stable piezomagnetic sensitive properties. 2 Higher magnetic elastic deformation, and lower magnetic plastic deformation. Therefore, research on the magntostricive property of FebacCu1Nb3Si15.5B7/Silicone rubber compound film have a very profound significant. In this experiment, the powder is made of amorphous films which are embrittled by atmosphere heat treatment. After that, by means of ethanol wet ball mills to prepare amorphous powder with the average grain size is about $20 \mu \mathrm{m}$. The resin matrix is mingled according a given ratio by $107 \#$ Silicone rubber and are silane ethyl. The powder and resin are treated by the process of matrix dense tried mixed, compression molding and ossifying at normal temperature to be formed this film [1]. We used Sigmar ASM2-1 portable precision strain gauge with $120 \Omega \pm 2 \%$ twin bridge strain film to test the magneto- elastic property of FebacCu1Nb3Si15.5B7/Silicone rubber compound film.

\section{Experiments}

Preparation of FebacCu1Nb3Si15.5B7/Silicone Rubber Compound Film. Making use of FebacCu1Nb3Si15.5B7 amorphous powder (molar ratio), which average particle size about $30 \mu \mathrm{m}$ and atmosphere heat treatment in $540^{\circ} \mathrm{C}$ for $0.5 \mathrm{~h}$. Matrix resin material: $107 \#$ silicone rubber and are silane ethyl mingled with the mass ratio of $9: 1$. The dilution resin material is acetone which takes $20 \%$ mass fraction of resin material. Resin material and amorphous powder are used with the mass ratio of 2:1, 1:1, 1:2, 1:3, 1:4, and 1:5 six types, which were stirred in a vacuum reaction kettle with a vacuum degree lower than $0.1 \mathrm{pa}$. After that, poured out the mixture from agitated reactor 
into a matrix and shaped up into $5 \times 10 \times 0.5 \mathrm{~mm}$ composite film, and solidified at NPT in 24 hours. Principle of Testing. By using ASM2-1 portable precision strain gauge to test the transformation of this composite film, and the principle of which is shown in Fig. 1:

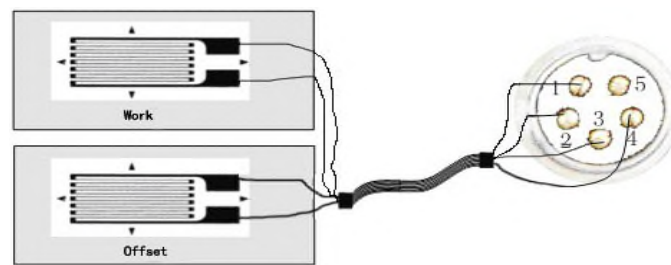

Fig. 1 Twin bridge strain test.

The testing details are that, it is used two plates of foil gauges which have similar elastic modulus as compound film, one is pressed into a film in the external magnetic field, and another is pressed into an equal film out of the magnetic field. The testing environments including pressure, temperature, humidity and residual stress, by which these two plates of films should be the same.

Before applying the external magnetic field, the strain gage have been zero set, and after that it will display accurately value of the strains in different magnetic fields.

Magnetostrictive Principle of Compound Film. In order to find the best film that has a good magnetostriction property, one should make it clear that what factors induce the advantage and disadvantage of the magnetostriction property of this type of film. We can assume that the influence factors are mainly connected with the contention of amorphous powder, the elasticity modulus, and poisson's ratio of amorphous powder \& silicon rubber, which induced the changes of composite films' elasticity modulus and poisson's ratio. These two factors are interacted on each other.

Firstly, the induction of magnetostriction in the composite film is the amorphous powder, since the applying magnetic field induced the powders be magnetized. The tiny particles would have some changes in length and volume. In addition, among the particles will also have some interactions in terms of inner stress which consist of grain stress FS, magnetic force with the direction of imposed magnetic field $\mathrm{FH}$, and shearing strength induced by rotation of domain in particles FM, which are shown in Fig. 2. These types of force induced the composite film come about a macroscopical magneto- striction effect. The inner stress of this compound film are different by numerical investigation. One can observe that, the stress distribution in powders is concentrated, and in rubber is uniform stress.

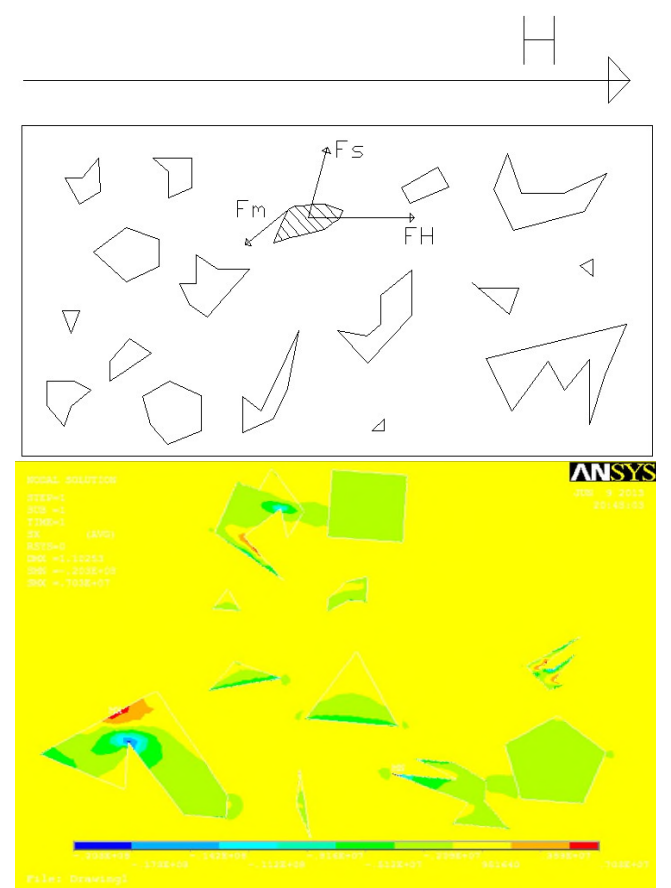

Fig. 2 Stress state of amorphous powder in rubber substrate. 
And then, the interior stress of this film is in proportion to the ratio of the powder. High magnetostrictive film have a higher content of amorphous powder. Meanwhile, the magnetostrictive also have business on elastic modulus of the film. Because elastic modulus is large different between amorphous powder and silicone rubber, and the total elastic modulus of composite film is resolved on the ratio of powder and rubber. In theory, elastic modulus of amorphous powder is far higher than silicone rubber, since films with low content of powder have a smaller elastic modulus than which with higher ones. In other words, film with low powder content is easy to be deformed and on the contrary, one with higher powder is hard to be deformed. So, these two factors which generate interactions obstruct each other, and the work must to find out an appropriate ratio between powder and silicone rubber which make the composite film have a best magnetostrictive effect.

Field Rationale of Magnetostrictive Film. In order to estimate a theoretical magnitude of magnetostriction property, a schematic model is made which is shown in Fig. 3.

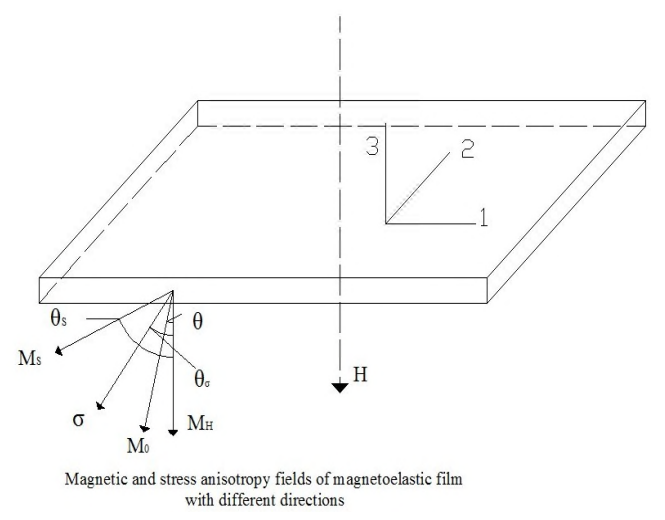

Fig. 3 Theoretical fields model in magnetostrictive film.

In Fig. 3, $\mathrm{H}$ represent applied magnetic field, $\mathrm{MH}$ is the direction of magnetization, $\sigma$ is the direction of stress, and MS is the direction of magnetostriction. The magnetoelastic film is assumed to have an uniaxial in plane magnetic anisotropy with its axis deviation angle of $\theta$ s with respect to the $\mathrm{H}$ direction. Angle $\theta$ represent a difference between magnetization which means the direction angle when magnetic field has not been applied and the applied magnetic field's direction. $\sigma 0$ represent the angle between stress and magnetic field.

To calculate the values of magnetostriction and saturation magnetostriction, LandauLifshitz-Gilbert equation [2] can be used to describe the spatial distribution and time evolution of magnetostriction $\mathrm{M}$.

$$
\frac{d M}{d t}=\frac{\gamma H_{e f f}}{\frac{1}{M}+\frac{\alpha}{M_{s}}}
$$

where, $\mathrm{M}$ is the magnetostriction, MS is saturation magnetostriction, Heff is the effective magnetic field, $\gamma$ is the gyromagnetic ratio, and $a$ is the Gilbert damping constant. The effect magnetic field can be written as

$$
H_{e f f}=H+H_{a}+H_{\sigma r}+H_{\sigma a}
$$

In Eq. 2, the effect magnetic field can be overlapped by four types of fields which can be calculated as follows separately:

The uniaxial anisotropy field is

$$
H_{a}=\frac{2 K_{u}}{\mu_{0} M_{s}^{2}} \overrightarrow{e_{a}}\left(\overrightarrow{e_{a}} \cdot M\right)=\frac{H_{k}}{M_{s}} \overrightarrow{e_{a}}\left(\overrightarrow{e_{a}} \cdot M\right)
$$

Here ea is the unit vector along the easy axis, $\mathrm{Ku}$ is the anisotropy constant and

$$
H_{k}=\frac{2 K_{u}}{M_{s} \mu_{0}}
$$


The residual stress effective field can be calculated as

$$
H_{\sigma r}=\frac{H_{\sigma 1}}{M_{S}} \overrightarrow{e_{\sigma r}}\left(\overrightarrow{e_{\sigma r}} \cdot M\right)
$$

where, eor is the unit vector in residual stress direction, $\lambda$ is the magnetostriction constant of magnetoelastic film, and

$$
H_{\sigma 1}=\frac{3 \lambda \sigma_{r}}{\mu_{0} M_{s}}
$$

Also the applied stress effective field can be written as

$$
H_{\sigma a}=\frac{H_{\sigma 2}}{M_{s}} \overrightarrow{e_{\sigma}}\left(\overrightarrow{e_{\sigma}} \cdot M\right)
$$

where, e $\sigma$ is the unit vector along the applied stress direction and $\mathrm{H} \sigma 2$ can be defined as

$$
H_{\sigma 2}=\frac{3 \lambda \sigma}{M_{s} \mu_{0}}
$$

Substituting Eqs (3)-(8) into Eq (2), and substituting Eq (2) into Eq (1), the value of magnetostriction $\mathrm{M}$ can be calculated

\section{Results and Discussion}

Mechanical Analysis of Amorphous Powder. The powder in the experiment is prepared by FebacCu1Nb3Si15.5B7 amorphous ribbon with mechanical comminution, and fluid energy milling to acquire thick powder which is shown in Fig. 4(a). After that, the thick powder has been wet ball milled with medium of alcohol for 24 hours. The powder after wet ball mill is much thinner which are observed in Fig. 4 (b). In the experiment, powder of thinner type has been used. These two types of powder have been analyzed by LPSA, which reveals the difference of size distribution of amorphous powder before and after wet ball milling. One can observe from figure that, the median particle diameter of thick powder is $168.68 \mu \mathrm{m}$ and size distribution is not homogeneous. The grain size concentrated in a narrow range, with the biggest grain size $341.69 \mu \mathrm{m}$.
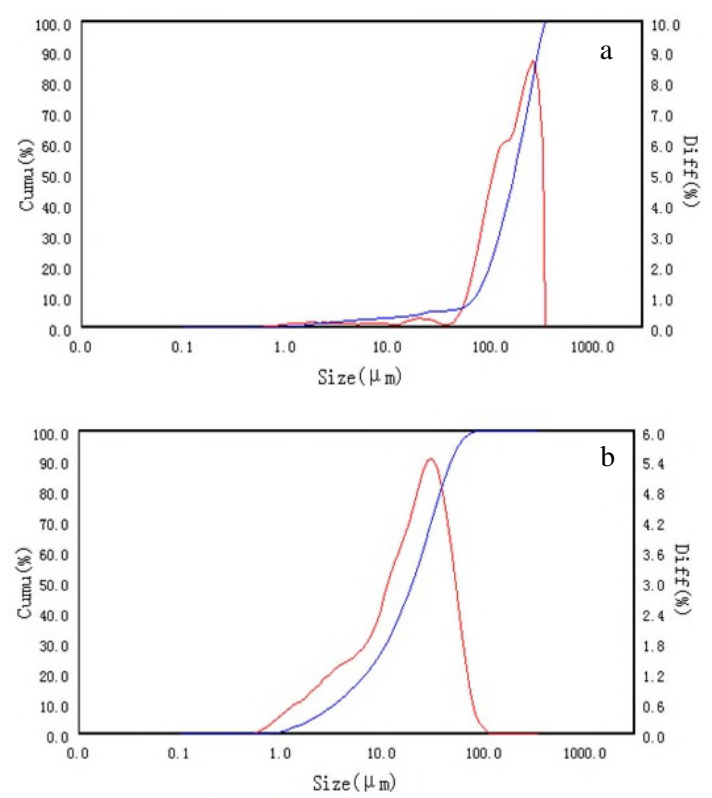

Fig. 4 Analyzed data before(a) and after(b) ball milling by LPSA.

The grain size of the powder after milling has a very large reduction. Size distribution range is more widely and appears normal distribution. The median particle diameter of thinner powder is 
$19.77 \mu \mathrm{m}$, and maximum size is $105.24 \mu \mathrm{m}$. The blue line in the figure represents volume fraction of grain changes with its size. And the red line represents diffusion degrees of grain in each dimension of the particles.

Surface Topography of Compound Film. Fig. 5 is four pictures of film with powder ratio at $75 \%$ in different magnifying power by the high of the depth of field optical microscope. Where Fig. 5(a), (b), (c) and (d) represents 500 times, 1000 times, 2000 times and 3000 times' magnified pictures separately. From the pictures one can perceive that, the particles have been scattered into the rubber equably by the stirring of agitated reactor. Maximum grain size is $100 \mu \mathrm{m}$ and average size is $19 \mu \mathrm{m}$ after ball milling. The powders are closely contacted with rubber matrix and can not be found any bubble. It's well bedded among amorphous powder, and inexistence the phenomenon of particle agglomeration in this film. But with the enhancement of powder, this phenomenon more or less cannot be ignored.

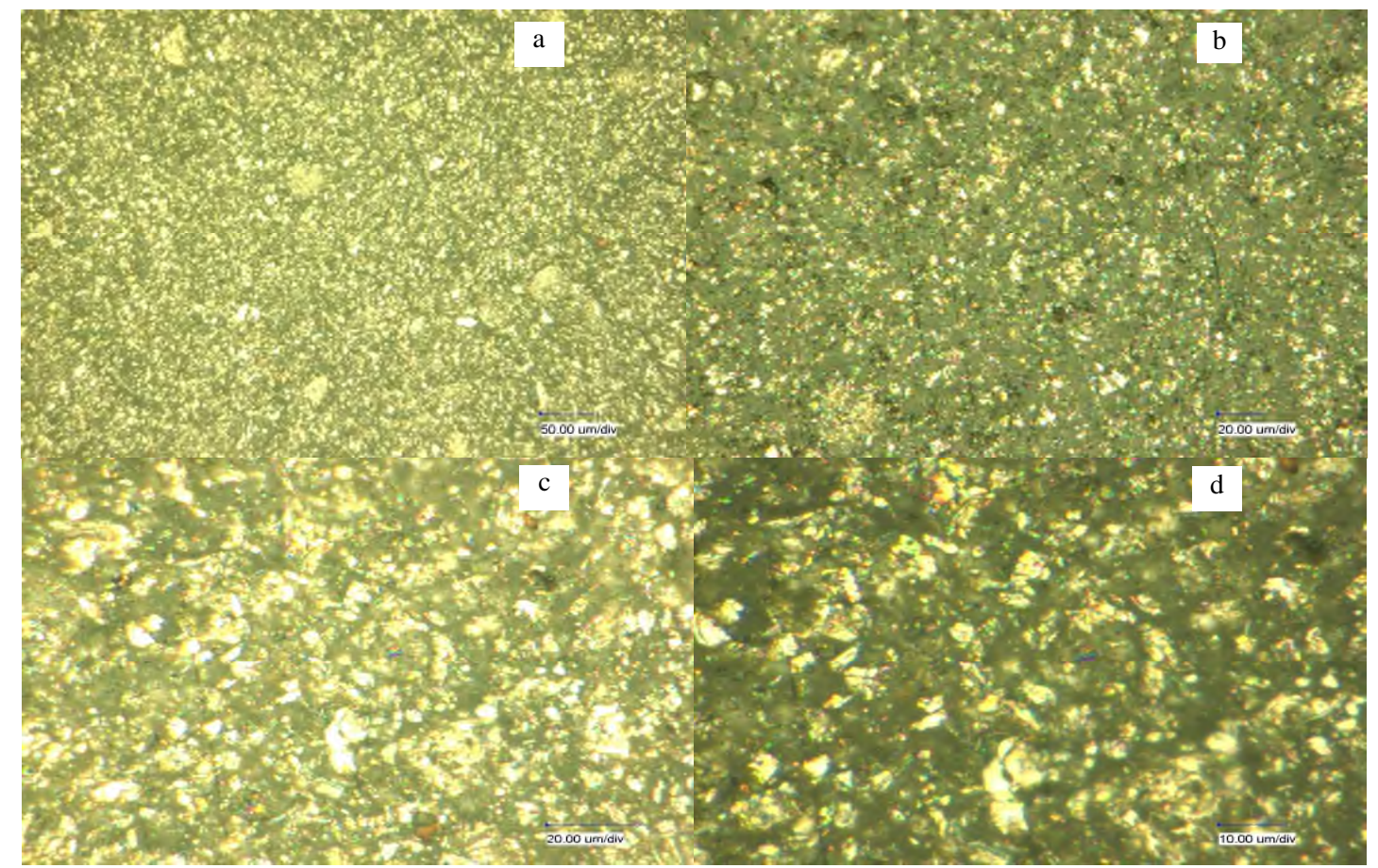

Fig. 5 Microscopic photos of amorphous powder $\mathrm{Wt} \%$ for $75 \%$ of compound film.

Magnetoelastic Properties of Composite Film. Fig. 6 displays a series of magnetic hysteresis elastic loops [3] in different magnetoelastic films with different quality ratio of amorphous powder.

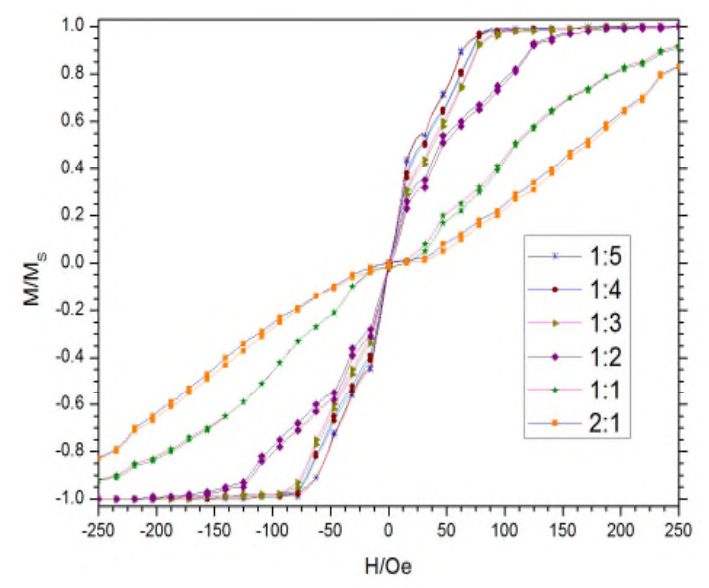

Fig. 6 Magnetic hysteresis on magnetostrictive films with different quality ratios of amorphous powder. 
In the experiment, the magnetic fields have been applied upload and offload on the ranges between -250 and 250 Oe, field step length is 15.625 Oe. It can be perceived from figure that the changes of magnetostriction with applied magnetic field.

In the mass, the property of magnetoelastic hysteresis is not obviously. It is vital for stress-impedance effect that whether the film has an excellent low magnetoelastic hysteresis property. However, in the figure, one can see that the film with lower powder ratio have a tiny magnetoelastic hysteresis phenomenon in a low magnetic field, and the phenomenon will reduce with the enhancement of powder ratio.

The saturation magnetostriction of compound film is related to applied field and mass ratio of amorphous powder. It will acquire saturation magnetostriction at a lower applied magnetic field with a higher mass ratio of powder. Further more, the magnetostriction of compound film also has some thing to do with addition of reinforcing agent in rubbers, since the addition of which may influence the elastic modulus and elastic deformation of the matrix silicon rubber

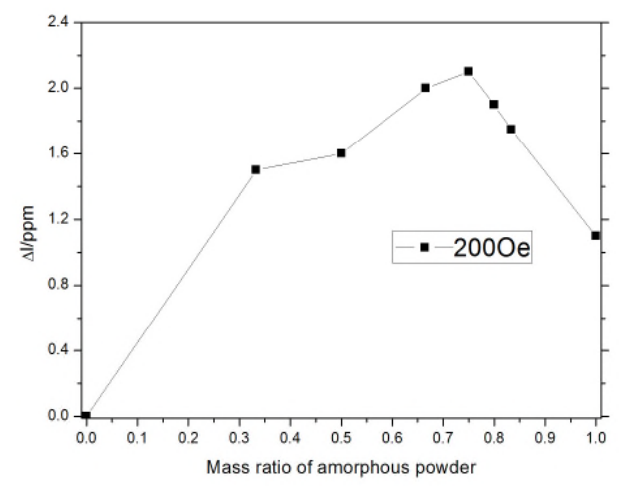

Fig. 7 Relationship between magnetic elastic variables and quality ratio of powder under the external magnetic field with 200 Oe.

Fig. 7 is the magnetostriction with the mass ratio of powder. We can see that the magnetostriction reaches a maximum value of $2.1 \mathrm{ppm}$ with the mass ratio at $75 \%$. Before the very ratio, magnetoelastic property increased with the addition of powder and not appears linear relation, but after which magnetoelastic changes with the addition of powder in a linear decrease. From the result of experiment, we can draw a conclusion that the elastic modulus of compound film and the mass ratio of powder is a group of mutual resistant factors. Although a film with low mass ratio of powder has a lower elastic modulus, the inner stress which induced by the magnetization of amorphous powder is smaller. And the film with high mass ratio of powder has a higher elastic modulus, but the inner stress induced by the magnetization of powder is bigger than the one with low mass ratio of amorphous powder. Therefore, when the powder content is $75 \%$ of mass ratio, the film reaches a balance point between inner stress and elastic modulus that makes the compound film has a best magnetostriction.

According to the generalized variational principle of elastic theory, the ranges of inner stress in different compound films with certain deformations can be estimated by principles of the most respect and the minimum potential energy. The ranges of elastic modulus with particle enhanced compound film Ec can be calculated by Eq. 9 and 10 [4].

$$
\frac{E_{p} E_{m}}{E_{m} v_{p}+E_{p} v_{m}} \leq E_{c} \leq \frac{1+\gamma_{m}+22\left(\lambda-2 v_{m}\right)}{1-\gamma_{m}-2 \gamma_{m}^{2}} E_{m m} v_{m}+\frac{1-\gamma_{p}+2 \lambda\left(\lambda-2 \gamma_{p}\right)}{1-\gamma_{p}-2 \gamma_{p}^{2}} E_{p} v_{p}
$$

In which $\lambda$ can be defined as

$$
\lambda=\frac{\gamma_{m}\left(1+\gamma_{p}\right)\left(1-2 \gamma_{p}\right) v_{m} E_{m}+\gamma_{p}\left(1+\gamma_{m}\right)\left(1-2 \gamma_{m}\right) v_{p} E_{p}}{\left(1+\gamma_{p}\right)\left(1-2 \gamma_{p}\right) v_{m} E_{m}+\left(1+\gamma_{m}\right)\left(1-2 \gamma_{m}\right) v_{p} E_{p}}
$$

where, the index $\mathrm{m}$ and $\mathrm{p}$ represent matrix rubber and powder, $\mathrm{E}, \gamma, \mathrm{v}$ represent elastic modulus, 
poisson's ratio and volume fraction of each component separately.

On the basis of physical parameter which is shown in Table 1 and Eqs (9), (10). We could easily confirm the range of elastic modulus on compound film with $75 \%$ powder mass ratio.

Table 1. Physical properties of amorphous powder and silicone rubber matrix [5].

\begin{tabular}{c|c|c|c|c|c}
\hline $\begin{array}{c}\text { Physical } \\
\text { index }\end{array}$ & $\begin{array}{c}\text { Elastic } \\
\text { modulus E }\end{array}$ & $\begin{array}{c}\text { Poisson's } \\
\text { ratio } \gamma\end{array}$ & $\begin{array}{c}\text { Volume } \\
\text { fraction }\end{array}$ & Density $\rho$ & Mass ratio \\
\hline $\mathrm{Fe}_{\mathrm{bac}_{\mathrm{Cu}} \mathrm{Cu}_{1} \mathrm{Nb}_{3} \mathrm{~S}}$ & $100 \mathrm{Gpa}$ & 0.28 & 1 & $7.87 \mathrm{~g} / \mathrm{cm}^{3}$ & 3 \\
$\begin{array}{c}\mathrm{i}_{15.5} \mathrm{~B}_{7} \text { powder } \\
\begin{array}{c}\text { Silicon rubber } \\
\text { matrix }\end{array}\end{array}$ & $6 \times 10^{-3} \mathrm{Gpa}$ & 0.46 & 3.015 & $0.87 \mathrm{~g} / \mathrm{cm}^{3}$ & 1 \\
\hline
\end{tabular}

Via calculating, the film's elastic modulus with $75 \%$ powder mass ratio Ec ranges between values $8.0 \times 10^{-3}$ and $24.93 \mathrm{Gpa}$. According to Fig. 7, the film reaches saturation magnetostriction value $2.1 \mathrm{ppm}$ with applied magnetic field of 200 Oe. Therefore, we can confirm its range of stress in cross section via elastic modulus and the value of deformation. As we know, the Hooke's law can be written as [4].

$$
F=\frac{A}{L} E \Delta L
$$

where, A, L and E represent cross area, length and elastic modulus separately. The dimension of the film is $\mathrm{L} \times \mathrm{W} \times \mathrm{H}=5 \times 10 \times 0.5 \mathrm{~mm}$. The Fmin and Fmax can also be written as: Fmin $=6 \times 103 \Delta \mathrm{L}$, Fmax $=108 \Delta$ L. The curves of Fmin and Fmax with applied field and strain have been made in Fig. (8).
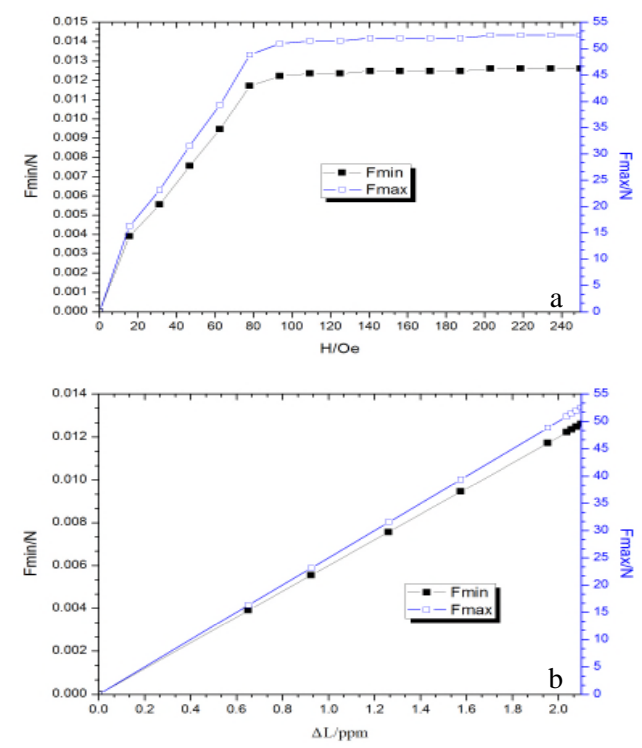

Fig. 8 The maximum and minimum values of stress with magnetic field (a) and strain (b).

In accordance with generalized variational principle, stress in cross section can be calculated. The actual stress with each deformation is more than the very value of Fmin and less than that of Fmax.

\section{Conclusions}

The film in the article has a good property in magnetostriction with low hysteresis. Amorphous powder has scattered in silicone rubber matrix evenly. The compound film is a kind of isotropy material and has a profound significant in stress sensitive sensors. Result shows, the elastic modulus is in proportion to the ratio of amorphous powder and have the best magnetostriction property with 
powder mass ratio at $75 \%$. We also have accurately calculated the range of stress in transverse by theory of generalized variational principle and Hooke's law in this paper.

\section{Acknowledgement}

This project was financially supported by the Fundamental Research Fund for the Youth Fund of Jiangxi academy of science (2017-YZDZ-10), and National Natural Science Foundation of China (51505203).

\section{References}

[1] Z. H. Zhu, A way of making force sensitive silastic film. Chinese patent: CN 1966550A, 2007-5-23.

[2] L. D. Lndau, E. M. Lifshitz, Electrodynamics of Continuous Media, J. Pergamon Oxford, 28 (1975) 0148-0151.

[3] N. Bayri, S. Atalay, In-andout-of-phase thermo mechanical fatigue of a Ni-based single-crystal superalloy, J. Alloys Compd. 381(1-2) (2004) 245-249.

[4] Y. X. Wang. Structural designment of composite material, M. Beijing: Chemical Industry Press, $2001,64$.

[5] METGLAS data sheet (2001), www.metglas.com.

[6] G. B. Ma. Failure behavior of the superalloy MAR-M247 LC under LCF. HCF and Combined LCF/HCF loading. Munich, J. Rare Met. Mater. Eng. 37(2) (2008) 286-288.

[7] J. A. Chen, W. Ding, Y. Zhou, Y. Cao, Fatigue behavior of the superalloy IN 713C under LCF-,HCF- and superimposed LCF/HCF-loading, J. Mater. Lett. 60 (2006) 2554-2557.

[8] Y. Zhou, W. Ding, X. H. Mao, Thermal fatigue of single-crystalline superalloy CMSX-4:a comparison of epitaxial laser-deposited material with the base single crystal, J. Thin Solid Films 489 (2005) 177-180.

[9] J. Hu, H. W. Qin, J. Chen, Y. Z. Zhang, Study on Performance of Self-adaptive Mechanical Seal under Variable Working Conditions, J. Magnet. Magn. Mater. 266 (2003) 290-295.

[10] K. Mohri, T. Uchiyama, L. P. Shen, Sens. High temperature erosion-oxidation of Cr3C2-NiCr thermal spray coatings under simulated turbine conditions, J. Corros. Sci. 91 (2001) 85-90.

[11] R. Juhasz, A. Cziraki, L. F. Kiss, A. Characterization of fretting wear of cobalt based superalloys at high temperature for aero-engine combustor components, J. Lovas, Mater. Sci. Eng. A 375-377 (2004) 1057-1061.

[12] I. Taher, M. Aslam, M. A. Tamor, T. J. Potter, R. C. Elder, Microstructure and properties of vacuum melting Cr3C2-NiCr coating by nickel-based brazing foil, J. Sens. Actuat. A. Phys. 45 (1994) 35 .

[13] A. F. Cobeno, A. Zhukov, J. M. Blanco, V. Larin, Studies on the dissolution characteristics of stainless steel base metal in the liquid nickel-based amorphous and crystalline brazing filler metals during vacuum brazing process, J. Gonzalez, Sens. Actuat. A. Phys. 91 (2001) 95.

[14] K. H. Shin, M. Inoue, K. I. Arai, Wear behavior of brazed WC/NiCrBSi(Co) composite Coatings J. Appl. Phys. 86(8) (1999) 5465. 